\title{
Optically detected spin-orbit torque ferromagnetic resonance in an in-plane magnetized ellipse
}

P. S. Keatley*, K. Chatzimpaloglou, T. Manago†, P. Androvitsaneas, T. H. J. Loughran, and R. J. Hicken Department of Physics and Astronomy, University of Exeter, Stocker Road, Exeter, EX4 4QL, UK †Department of Applied Physics, Fukuoka University, 8-19-1 Nanakuma, Jonan, Fukuoka 814-0180, Japan

G. Mihajlović, L. Wan, Y.S. Choi, and J. A. Katine

Western Digital Research Center, Western Digital Corporation, San Jose, California 95119, USA

*Corresponding author e-mail: p.s.keatley@exeter.ac.uk

Time-resolved scanning Kerr microscopy has been used to perform optically-detected, phaseresolved spin-orbit torque ferromagnetic resonance (SOT-FMR) measurements on a microscale CoFeB ellipse at the centre of a Pt Hall cross subject to RF and DC current. Time-resolved polar Kerr images revealed localized dynamics with large amplitude at the center, and weaker amplitude at the edges. Therefore, field swept SOT-FMR spectra were acquired from the so-called center mode to probe the SOTs active at the center of the ellipse, thus minimising non-uniform edge contributions. When the magnetic field was applied at $30^{\circ}$ from the hard axis of the ellipse and a DC current applied, a marked asymmetry was observed in the amplitude and linewidth of the FMR peaks as the applied field was reversed. Both absorptive and dispersive parts of the spectra were in good agreement with a macrospin calculation. The damping parameter $(\alpha)$ and the Slonczewski torque parameter (ST) were determined to be 0.025 and $(6.75 \pm 0.75) \times 10^{-7} \mathrm{Oe}^{-1} \mathrm{~cm}^{2}$ respectively. The hard axis SOT-FMR linewidth was found to be almost independent of the DC current value, suggesting that the SOT has minimal influence in the hard axis configuration and also that thermal effects were insignificant. This study paves the way to spatially-resolved measurements of SOT probed using localized modes of microscale devices that go beyond the spatially averaged capability of electrical measurement techniques. 
Following the prediction ${ }^{1}$ and demonstration ${ }^{2}$ of spin transfer torque (STT) more than two decades ago, related phenomena such as spin pumping ${ }^{3,4,5}$, spin accumulation ${ }^{6,7,8}$, and the spin Hall effect $(\mathrm{SHE})^{9,10,11,12,13}$ in metals have attracted great attention due to potential applications in magnetic random access memory (MRAM). In the SHE, the efficient generation of pure spin currents at the interface between a ferromagnet and a heavy metal such as platinum, tantalum, or tungsten results from the strong spin-orbit coupling in the heavy metal (HM). So-called spin-orbit torque (SOT) devices take advantage of the SHE to reduce device size, improve energy efficiency, and increase device longevity since the write current no longer needs to pass through the delicate tunnel barrier of a STT-MRAM cell, but instead remains predominantly in the HM underlayer.

Recent studies of SOTs in HM/FM bilayer devices have focused on FM elements with perpendicular magnetic anisotropy (PMA) $)^{9,10,11,12}$. However, for STT-MRAM applications, it has been reported that in-plane magnetized SOT devices have potential for lower energy consumption, faster switching times, and enhanced switching probability ${ }^{14}$. In-plane SOT devices ${ }^{15,16}$ also permit the use of a greater variety of ferromagnetic materials since they are not limited to the few choices that yield PMA. On the other hand, in-plane magnetised elements can support non-uniform equilibrium states, such as so-called leaf, $\mathrm{S}$, and $\mathrm{C}$ states ${ }^{17}$ where the magnetization cants along edges of the element that are not parallel to the applied magnetic field. The corresponding non-uniform internal field allows spatially localized modes to be supported such as the so-called center and edge modes. ${ }^{18}$ Since the Slonczewski torque term in the Landau-Lifshitz-Gilbert equation of motion depends upon the local magnetization ${ }^{19}$, a non-uniform equilibrium state can lead to a non-uniform torque across the element. In electrical measurements only the spatially averaged orientation of the magnetization is probed by magnetoresistance or Hall effect measurements, while the voltage rectification within the device does not permit the phase of the dynamic response to be determined. However, recently developed optical ${ }^{19,16,20,21,22}$ and $x$-ray ${ }^{23}$ techniques are now able to directly probe the local magnetization within SOT devices.

Here time-resolved scanning Kerr microscopy ${ }^{24}$ (TRSKM) has been used to perform opticallydetected, phase-resolved SOT induced ferromagnetic resonance (SOT-FMR) measurements in which the center localized magnetization dynamics of a microscale SOT device were directly probed with a spatial resolution of $\sim 400 \mathrm{~nm}$. Field swept SOT-FMR spectra were acquired from a $2 \times 0.8 \mu \mathrm{m}^{2} \mathrm{in}$ plane magnetized $\mathrm{CoFeB}(2 \mathrm{~nm})$ ellipse deposited on a $\mathrm{Pt}(6 \mathrm{~nm})$ underlayer as a function of the applied in-plane field angle. Optically detected SOT-FMR has previously been used to characterize the torque on the magnetization due to an RF current in spin Hall nano-oscillator devices ${ }^{19}$. In the present work, an elliptical element was subject to the Oersted (Oe)-field and SOT, generated by the sum of both an RF current $\left(I_{\mathrm{RF}}\right)$ and a DC current $\left(I_{\mathrm{DC}}\right)$. Field swept SOT-FMR spectra were recorded at the centre of the ellipse so that the center mode could be utilised as a probe of the SOTs at the center of the ellipse, thus avoiding non-uniform edge effects. The dependence of the optically detected spectra upon the orientation of the in-plane applied field was well reproduced by a macrospin model, allowing the values of both the damping parameter and the SOT parameter to be determined.

The layout of the device and the experimental set-up are shown in Figure 1. Additional details of the device fabrication and characterisation have been reported previously ${ }^{14}$. Magnetron sputtering was used to deposit a Pt(6)/ $\mathrm{CO}_{40} \mathrm{Fe}_{40} \mathrm{~B}_{20}(2) / \mathrm{MgO}(1) / \mathrm{TaOx}$ (4) (thicknesses in $\mathrm{nm}$ ) film onto a Si substrate with a $\mathrm{SiO}_{2}(250 \mathrm{~nm})$ thermally oxidised overlayer. The stack was then annealed, after which electron beam lithography and ion milling were used to form a Hall cross device consisting of a $2 \times 0.8 \mu \mathrm{m}^{2}$, $\mathrm{CoFeB} / \mathrm{MgO} / \mathrm{TaO}$ x ellipse centred on a $\mathrm{Pt}(6 \mathrm{~nm})$ cross. Outside the perimeter of the ellipse, the Pt was intentionally overmilled by approximately $1 \mathrm{~nm}$. 

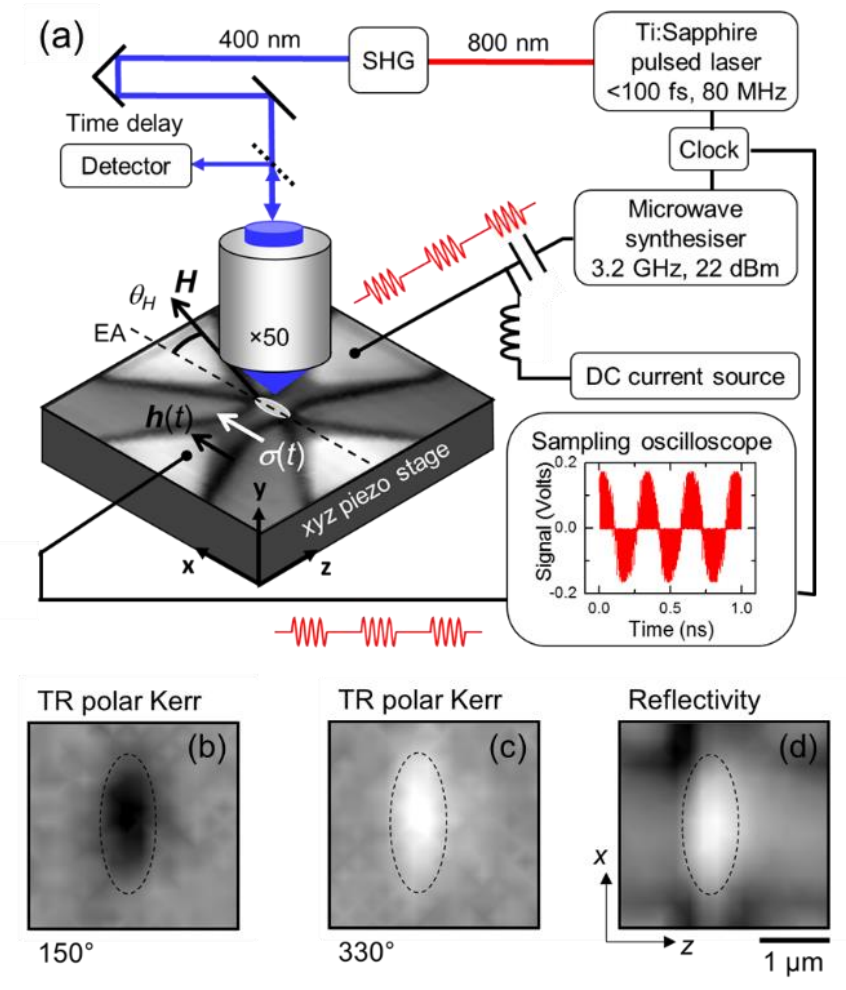

Figure 1 (a) Optically-detected, phase-resolved SOT-FMR in a TRSKM. Ultrafast laser pulses with duration $<100 \mathrm{fs}, 80-\mathrm{MHz}$ repetition rate, and $800-\mathrm{nm}$ wavelength are converted to $400-\mathrm{nm}$ wavelength by SHG. The pulses are synchronized with the GHz-frequency RF output of a microwave synthesiser. The phase of the RF waveform was monitored using a sampling oscilloscope (inset, measured waveform showing amplitude modulation). RF and DC current was passed to the device simultaneously via a bias tee, high frequency microscale coaxial probes, and the wider $(2 \mu \mathrm{m})$ current lead of the Pt Hall cross. The width of the current lead was approximately equal to the length of the long, easy axis of the ellipse, which was perpendicular to the current direction. The resulting Oe-field $(\boldsymbol{h}(t))$ and injected spin polarisation $(\sigma(t))$ at the Pt/CoFeB interface were hence parallel to the easy axis, from which the in-plane applied field $\boldsymbol{H}$ angle $\theta_{H}$ is also defined. (b), (c) TR polar Kerr images acquired at successive antinodes of precession of a center-localized mode show large opposite (black, white) contrast at the center of the ellipse as the magnetization cants in and out of plane, and weaker contrast at the edges. (d) Reflectivity image of the ellipse. The outline of the ellipse is indicated by a dashed line within each image.

A diffraction-limited spatial resolution of $\sim 400 \mathrm{~nm}$ was achieved by reducing the wavelength of the probing laser pulses from $800 \mathrm{~nm}$ to $400 \mathrm{~nm}$ by means of second harmonic generation (SHG) ${ }^{25,26}$. A filter was used to remove residual $800 \mathrm{~nm}$ light and the beam was expanded $(\times 5)$ to reduce its divergence. The beam was then linearly polarised and attenuated to an average power $\sim 200 \mu \mathrm{W}$ before being focused by a $\times 50$ (N.A. 0.55$)$ long working distance $(\sim 11 \mathrm{~mm})$ microscope objective lens. A 4 ns optical delay line was used to acquire time-resolved signals and accurately set the phase of the probe laser pulse with respect to the RF current passing through the device.

The RF current was generated by a microwave synthesiser with RF power of $22 \mathrm{dBm}$ and frequency of $3.2 \mathrm{GHz}=n \times 80 \mathrm{MHz}$, where $n=40$, and $80 \mathrm{MHz}$ is the synchronous repetition rate of the laser. The RF current amplitude was $8 \mathrm{~mA}$ after accounting for the reflection coefficient of 0.87 at the device due to the transition from $50 \Omega$ coaxial cable and probes to the $700 \Omega$ device load. The current flowed in the wider $2 \mu \mathrm{m}$ current lead perpendicular to the long, easy axis (EA) of the ellipse 
so that the injected spin polarisation and Oe-field were parallel to the EA. The RF and DC Oe-fields acting on the ellipse were calculated to be approximately 25 Oe and 30 Oe (for $I_{D C}=10 \mathrm{~mA}$ ) respectively. Non-linear effects were not observed in either the measured or calculated spectra, and measurements were found to be insensitive to resonance thermal effects ${ }^{27}$. The RF current amplitude was modulated at $\sim 3.14 \mathrm{kHz}$ and the resulting modulation of the out-of-plane component of the dynamic magnetization detected via the polar Kerr effect, using a balanced polarizing photodiode bridge detector, and a lock-in amplifier. Amplitude modulation was used in analogy to electrical SOT-FMR measurements. ${ }^{19}$ The DC current was generated by a precision current source and combined with the RF current using a bias tee.

Macrospin calculations of the field swept SOT-FMR spectra were based on the model described in Ref. ${ }^{19}$, but extended to include both the SOT and Oe-field generated by both RF and DC currents. Following reference ${ }^{28}$, the Landau-Lifshitz Gilbert (LLG) equation of motion for the magnetization in the presence of Slonzcewski (in-plane) and field-like (out-of-plane) spin transfer torque terms may be written as

$\frac{d \hat{\mathbf{s}}}{d t}=\gamma \hat{\mathbf{s}} \times \mathbf{H}_{\mathrm{eff}}+\alpha \hat{\mathbf{s}} \times \frac{d \hat{\mathbf{s}}}{d t}+\gamma S T J \hat{\mathbf{s}} \times(\hat{\mathbf{s}} \times \widehat{\boldsymbol{\sigma}})+\gamma F T J \times \widehat{\boldsymbol{\sigma}},(1)$

where $\hat{\boldsymbol{s}}$ and $\widehat{\boldsymbol{\sigma}}$ are unit vectors parallel to the magnetization and injected spin polarization respectively, $J$ is the total current density, $S T$ and $F T$ are the amplitudes of the Slonzcewski and fieldlike torque terms, and $\gamma=g \mu_{\mathrm{B}} / \hbar$, where $g$ is the spectroscopic splitting factor, $\mu_{\mathrm{B}}$ is the Bohr magneton (and has negative sign), and $\hbar$ is Planck's constant divided by $2 \pi . \quad \mathbf{H}_{\text {eff }}$ is the effective magnetic field and includes contributions from the applied in-plane magnetic field, the uniaxial shape anisotropy field, the demagnetizing field, and the Oe-fields generated by the DC and RF currents passing through the device. Quasi-alignment of the magnetization with the applied field was not assumed in order to provide an accurate description of measurements made at applied field values comparable to the uniaxial anisotropy field. Instead the orientation of the equilibrium magnetization was determined from the solution of the time-independent part of equation (1) after linearization. It was therefore not possible to obtain explicit expressions for the linewidth of the field swept resonance. Further details of the macrospin model are presented in the supplementary material.

Preliminary time-resolved measurements and images were acquired to determine the conditions (frequency and field) for SOT-FMR and are presented in the supplementary material. TR polar Kerr images acquired at resonance, see Fig. 1(b-c) and supplementary material, show that the dynamic magnetization corresponds to a center-localized mode. The laser spot was then positioned at the centre of the ellipse to acquire optically-detected, phase-resolved SOT-FMR spectra corresponding to the center mode, so as to investigate SOTs active at the center of the ellipse, thus minimising nonuniform edge contributions. Figure 2 shows experimental spectra (symbols) acquired for different orientations of the in-plane applied magnetic field. The time-delay between the RF current and the probing laser pulse was set so that the laser pulse probed the absorptive (rather than the dispersive) component of the dynamic magnetic susceptibility. The dispersive component is presented in the supplementary material. Calculated spectra are overlaid (solid red lines) and show good agreement with the experimental data, confirming that the response of the center mode can be described as a single macrospin. When the field was applied along the EA $\left(\theta_{H}=0^{\circ}, 180^{\circ}\right)$, FMR peaks were not observed because no torque is exerted on the magnetization when the Oe-field and spin polarisation lie parallel to the equilibrium magnetization. In contrast when the field is applied along the hard axis $\left(\mathrm{HA}, \theta_{H}=90^{\circ}, 270^{\circ}\right)$, FMR peaks are clearly observed as the Oe-field and injected spin polarization exert maximum torque. However, no discernible asymmetry with respect to field polarity was 
observed in either the amplitude or linewidth of the HA FMR peaks as $I_{D C}$ was increased from 0 to 10 $\mathrm{mA}$ suggesting that SOTs are weak or inactive when the ellipse is magnetized along the HA, see supplementary material. Moreover, insensitivity of the HA linewidth to the DC current suggests that the measurements are insensitive to resonance thermal effects for $I_{\mathrm{DC}}$ up to $10 \mathrm{~mA}$, the maximum value applied in this work.

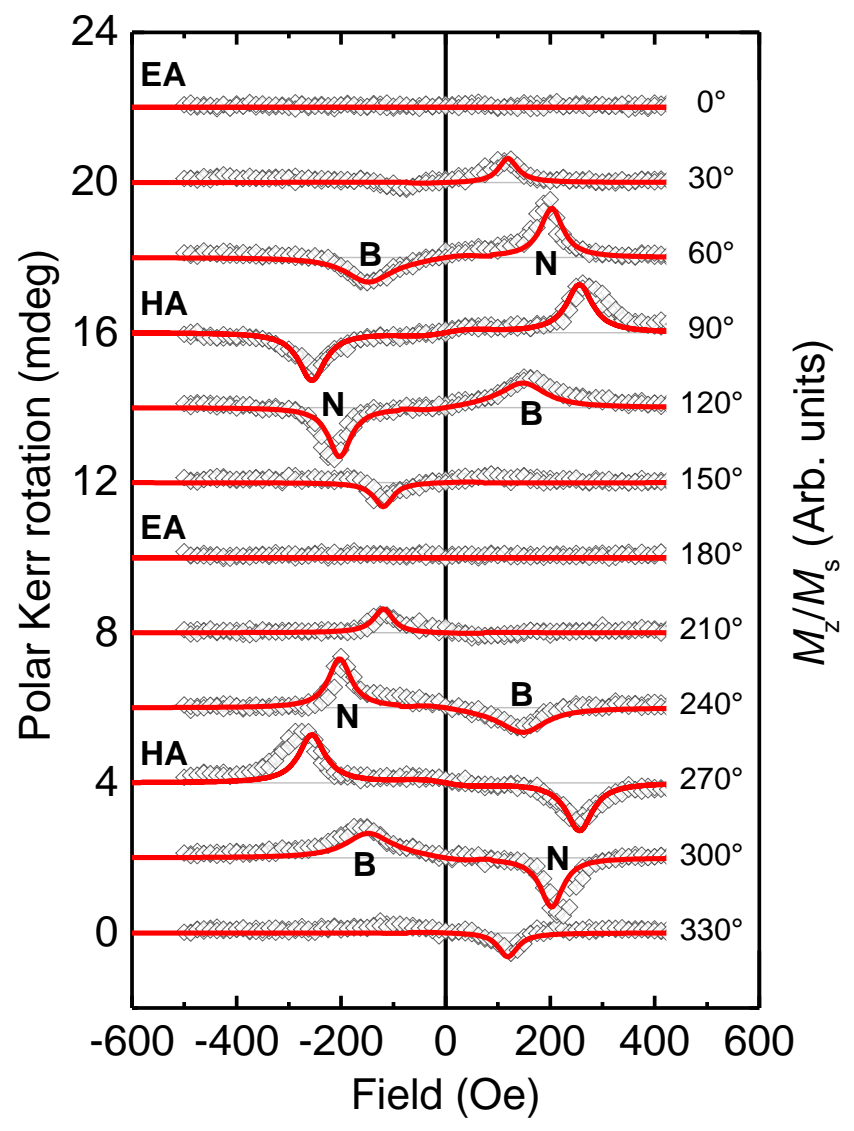

Figure 2. Optically-detected, phase-resolved SOT-FMR spectra (grey open symbols) corresponding to the imaginary component of the dynamic susceptibility and acquired over the full range of azimuthal angle $\theta_{\mathrm{H}}$ defined with respect to the EA $\left(0^{\circ}\right)$. $f_{\mathrm{RF}}$ and $\mathrm{ICC}_{\mathrm{DC}}$ had values of $3.2 \mathrm{GHz}$ and $10 \mathrm{~mA}$ respectively. Calculated spectra for a single macrospin are overlaid (red curves). The calculations assumed $f_{\mathrm{RF}}=3.2 \mathrm{GHz}, I_{\mathrm{DC}}=10 \mathrm{~mA}, I_{\mathrm{RF}}$ $=8 \mathrm{~mA}, \mathrm{ST}=6.75 \times 10-7 \mathrm{Oe} \mathrm{A}^{-1} \mathrm{~cm}^{2}$, a uniaxial anisotropy field of $90 \mathrm{Oe}$, demagnetizing field $=7500 \mathrm{Oe}, g$-factor $=$ 2.05, $\alpha=0.025$, Pt lead width $=3.5 \mu \mathrm{m}$, and Pt thickness $=6 \mathrm{~nm}$. The measured spectra are shown for both field sweep directions, while for clarity the calculated spectra are shown only for a single sweep direction (avoiding field sweep segments for which the energy minimisation routine can yield metastable equilibrium states after sweeping through remanence). The measured (calculated) spectra have been offset by 2 mdeg $\left(0.08 \mathrm{M}_{\mathrm{z}} / \mathrm{M}_{\mathrm{s}}\right)$ for clarity. Example broad and narrow FMR peaks are labelled $\mathrm{B}$ and $\mathrm{N}$ respectively.

The value of the damping parameter $\alpha$ was first determined from the HA linewidth for the case $I_{D C}=0 \mathrm{~mA}$ where the linewidths within the SOT-FMR spectra are found to be insensitive to the ST parameter. The linewidth is defined as the full width at half maximum of a Lorentzian curve fitted to the peaks in the calculated and experimental SOT-FMR spectra. Figure 3(a) shows the linewidth of the calculated spectrum for different values of the damping parameter. The average linewidth determined from experimental spectra measured for two different field histories (horizontal blue 
line) agree best with the calculations for $\alpha=0.030 \pm 0.003$, compared to $\alpha=0.035$ reported for smaller $\left(80 \times 205 \mathrm{~nm}^{2}\right)$ devices of the same composition ${ }^{14}$.

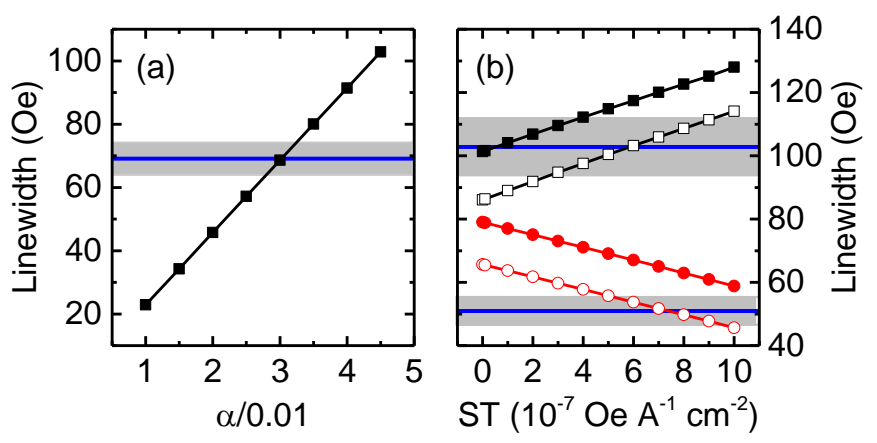

Figure 3. (a) Calculated HA linewidth (black squares) versus damping parameter $\alpha$ for $I_{D C}=0$. The horizontal blue line shows the average value measured for different field histories while the light grey band represents their standard deviation. (b) Calculated linewidth versus ST parameter for the broad (B) (black squares) and narrow $(\mathrm{N})$ (red circles) peaks at $+H$ and $-H$ respectively for $\theta_{H}=120^{\circ}$ and $I D C=10 \mathrm{~mA}$ (see Fig. 2). The filled (open) symbols assume $\alpha=0.030(=0.025)$. Comparison is made with the linewidth determined from 8 equivalent measurements of both the $B$ and $N$ peaks. The blue horizontal lines indicate the average experimental linewidth values, while the light grey band represents their standard deviation.

When the applied field orientation lies between the $E A$ and $H A$, and for $I_{D C}=10 \mathrm{~mA}$, both experimental and calculated spectra in Figure 2 show a marked asymmetry in the amplitude and linewidth of the FMR peaks at positive and negative field values. At these intermediate field angles, the equilibrium magnetization has a component that lies either parallel or antiparallel to the Oe-field and spin polarization, depending upon the field polarity. This leads to either an enhancement or reduction of the effective damping and corresponding SOT-FMR linewidth as most clearly observed in Figure 2 at angles $\pm 30^{\circ}$ from the HA. For example, broad (B) and narrow $(\mathrm{N})$ peaks are observed for $-H$ and $+H$ values at $\theta_{H}=60^{\circ}$, while B and $\mathrm{N}$ peaks occur for $+H$ and $-H$ values at $120^{\circ}$.

Spectra calculated for ST = 0, but including the Oe-field due to the DC current (not shown), also show asymmetry in the amplitude and linewidth with respect to field polarity similar to that observed in the experiment. Therefore, the asymmetry observed in the experiment is due to the combined effect of Oe-field and SOT. Changing the heavy metal from Pt to e.g. W, could confirm this conclusion since spin Hall angles of opposite sign are observed for these materials ${ }^{13}$.

The dependence of the resonance field and linewidth upon the orientation of the applied field is plotted for both positive and negative field polarity in Figure 4. The variation of the resonance field can be described as the superposition of a two-fold $\sin ^{2}\left(2 \theta_{\mathrm{H}}\right)$ term due to the uniaxial shape anisotropy and a one-fold $\cos ^{2}\left(\theta_{H}\right)$ term due to the DC Oe-field. The latter term results in a difference in resonance field with respect to field polarity for certain angles, and causes the values of the resonance field to differ between $\theta_{\mathrm{H}}=60^{\circ}$ and $120^{\circ}$, whereas these values would be equivalent in the absence of the Oe field (grey arrows highlight the difference in Figure 4(a)).

To obtain the best agreement between the resonance fields of the calculated and experimental SOTFMR spectra it was necessary to increase the assumed width of the current lead from $2 \mu \mathrm{m}$ to 3.5 $\mu \mathrm{m}$. This increases the area through which the current flows and reduces the current density and Oe field at the ellipse. Separate finite element simulations suggest that the DC current may spread out 
into the perpendicular Hall leads that are positioned at either end of the ellipse and perpendicular to the current direction. Spreading of the RF current was previously inferred within spin Hall nanooscillators ${ }^{19}$, due in part to the reactance of the device structure. It is therefore quite difficult to establish the current distribution within the device.

Calculated spectra (not shown) indicate that the resonance field has negligible dependence upon the ST parameter, so to observe the influence of SOT it is necessary to look in detail at either the amplitude or linewidth of the observed FMR peaks. Figure 4(b) shows that there is a distinct crossover in the linewidth value as the field angle crosses the HA and as the component of magnetization that is collinear with the Oe-field and spin polarization changes sign.
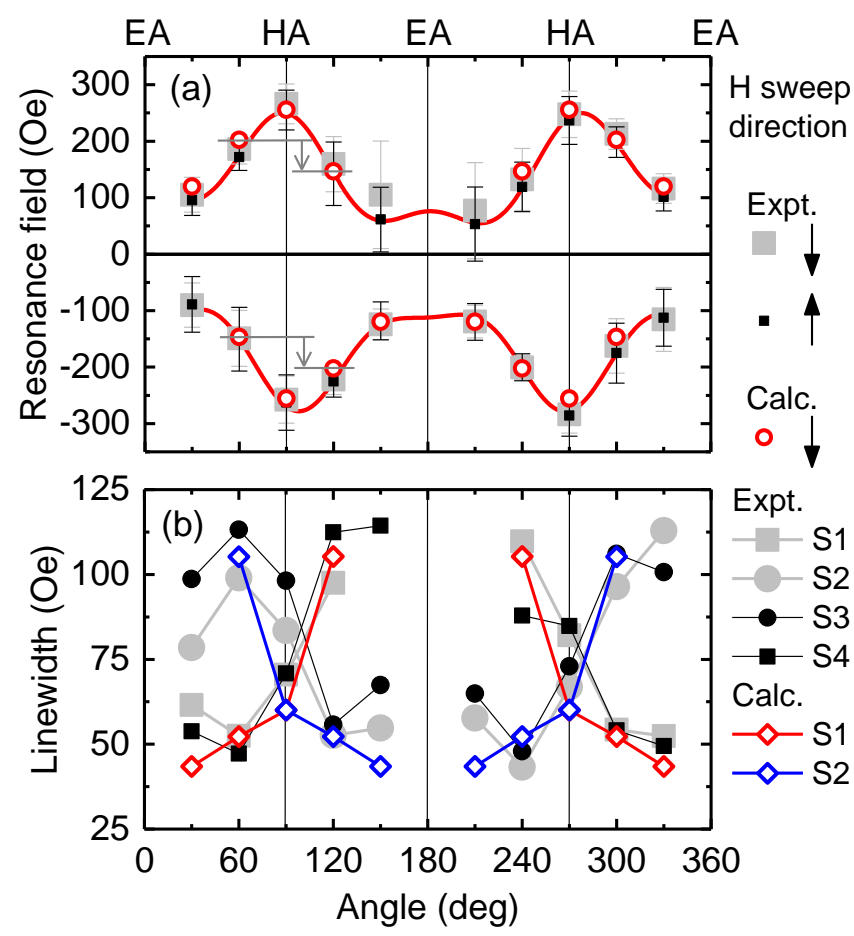

Figure 4. (a) Measured resonance field versus applied field angle for $I_{D C}=10 \mathrm{~mA}$. The field was swept from positive to negative values (large filled grey squares, down arrow) and then from negative to positive (small black filled squares, up arrow). The length of the error bar indicates the measured linewidth. The resonance fields determined from the calculated spectra assuming $\alpha=0.025$ and ST $=6.75 \times 10-7 \mathrm{Oe} \mathrm{A}^{-1} \mathrm{~cm}^{2}$ are also shown (open red circles). The guide to the eye (red curves) contains $\sin ^{2}\left(2 \theta_{\mathrm{H}}\right)$ and $\cos ^{2}\left(\theta_{\mathrm{H}}\right)$ terms, while the difference in the resonance field at an angle $30^{\circ}$ either side of the HA $\left(\theta_{\mathrm{H}}=90\right)$ is shown by small grey arrows. (b) Measured linewidth versus applied field angle (grey and black symbols), for different segments of the field sweep; positive to remanence (S1), remanence to negative (S2), negative to remanence (S3), remanence to positive (S4). The linewidths of the calculated curves are shown for segments S1 and S2 only for clarity.

In light of the symmetry of the anisotropy and Oe fields, the spectra for $\theta_{H}=60^{\circ}, 120^{\circ}, 240^{\circ}$ and $300^{\circ}$ are equivalent, each containing one $\mathrm{B}$ and one $\mathrm{N}$ peak with similar resonance fields and linewidths. It is therefore sufficient to compare these measured $\mathrm{B}$ and $\mathrm{N}$ linewidths with those calculated for a single field angle of $\theta_{H}=120^{\circ}$. Using the value $\alpha=0.030$ determined from the HA measurement, spectra were first calculated for different values of ST and the $B$ and $N$ linewidths extracted. Figure $3(b)$ shows a clear linear increase (decrease) of the linewidth of the $B(N)$ peaks as the ST parameter is increased from 0 to $10 \times 10^{-7} \mathrm{Oe}^{-1} \mathrm{~cm}^{2}$. Comparison is made with the 8 
equivalent measurements (field swept up and down for $\theta_{\mathrm{H}}=60^{\circ}, 120^{\circ}, 240^{\circ}$ and $300^{\circ}$ ) of both the B and $\mathrm{N}$ peaks. The blue horizontal lines indicate the average of the 8 experimental linewidth values.

Assuming $\alpha=0.03$, the calculated linewidth of the B peak for $\theta_{H}=120^{\circ}$ shows best agreement with the average measured linewidth (blue line) for $S T=0.6 \times 10^{-7} \mathrm{Oe} A^{-1} \mathrm{~cm}^{2}$. In contrast, a value of ST $>10 \times 10^{-7} \mathrm{Oe} \mathrm{A}^{-1} \mathrm{~cm}^{2}$ is needed to bring the calculated linewidth of the $\mathrm{N}$ peak into agreement with the measured values. However, if a smaller value of $\alpha=0.025$ is assumed, good agreement between

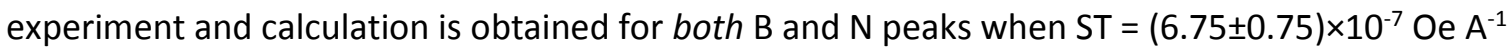
$\mathrm{cm}^{2}$. Comparable values of the ST parameter have been obtained from quasi-static measurements of the out-of-plane deflection in response to a modulated DC current ${ }^{29}$. Presumably the larger value of $\alpha=0.030$ determined from the HA spectra when $I_{D C}=0 \mathrm{~mA}$ is an overestimate, suggesting that the HA linewidth is more strongly affected by extrinsic relaxation mechanisms such as two-magnon scattering, and/or a more non-uniform equilibrium state. Measurements of the field-swept linewidth at different frequencies would allow the different contributions to the damping to be better understood ${ }^{30}$.

Angular dependent SOT-FMR measurements performed for $I_{D C}=1 \mathrm{~mA}$ do not show the marked asymmetry in amplitude and linewidth seen for measurements with $I_{D C}=10 \mathrm{~mA}$, see supplementary material. When the field was applied parallel to the $H A$ with $I_{D C}=1 \mathrm{~mA}$, comparison with macrospin calculations yielded a value for the damping parameter of $\sim 0.033$, while the expected variation of the linewidth due to the SOT was found to lie within the experimental uncertainty. The fact that the value of $\alpha$ decreased somewhat as $I_{D C}$ was increased from $1 \mathrm{~mA}$ to $10 \mathrm{~mA}$ suggests increased spatial uniformity of the equilibrium state and rules out a significant role for thermal effects up to $I_{D C}=10$ $m A$. For $I_{D C}=1 \mathrm{~mA}$, the calculated dependence of the linewidth upon the field orientation was found to be smaller than the experimental uncertainty and so only the measurements made at $I_{D C}=$ $10 \mathrm{~mA}$ were used to determine the value of the ST parameter.

In summary TRSKM has been used to perform optically-detected, phase-resolved SOT-FMR measurements. A center-localized mode was used to probe the SOTs active at the center of a microscale ellipse, thus avoiding non-uniform edge effects. Macrospin calculations reveal that a combination of both Oe-field and SOT are required to reproduce the marked asymmetry of the FMR spectra with respect to the polarity of the applied field. By comparing the measurements to the calculations, the values of the damping parameter and ST parameter can be determined, while the insensitivity of the measured $\mathrm{HA}$ linewidth to $I_{\mathrm{DC}}$ suggests that thermal effects do not influence the spectra for $I_{D C}$ values up to $10 \mathrm{~mA}$. The use of TRSKM as a direct probe of localized modes of microscale devices in SOT-FMR measurements goes beyond the spatially averaged capability of popular electrical measurement techniques and paves the way towards spatially resolved measurements of SOT.

The authors gratefully acknowledge the support of the Engineering and Physical Sciences Research Council under Grant Ref. EP/P008550/1. The data that support the findings of this study are openly available in Open Research Exeter (ORE), reference number [reference number TBC].

\section{Supplementary Material}

The supplemental material contains details of the preliminary time-resolved Kerr measurements and imaging, the phase-resolution of optically detected SOT-FMR and the dependence on DC current, insitu vector-resolved Kerr magnetometry, the macrospin model, and a comparison of TRSKM with other techniques. 
1. J. C. Slonczewski, Journal of Magnetism and Magnetic Materials 159 (1-2), L1-L7 (1996).

2. J. A. Katine, F. J. Albert, R. A. Buhrman, E. B. Myers and D. C. Ralph, Physical Review Letters 84 (14), 3149-3152 (2000).

3. Y. Tserkovnyak, A. Brataas and G. E. W. Bauer, Physical Review Letters 88 (11), 117601 (2002).

4. M. K. Marcham, L. R. Shelford, S. A. Cavill, P. S. Keatley, W. Yu, P. Shafer, A. Neudert, J. R. Childress, J. A. Katine, E. Arenholz, et al., Physical Review B 87 (18) (2013).

5. J. Li, L. R. Shelford, P. Shafer, A. Tan, J. X. Deng, P. S. Keatley, C. Hwang, E. Arenholz, G. van der Laan, R. J. Hicken, et al., Physical Review Letters 117 (7) (2016).

6. Y. Otani and T. Kimura, Philos. Trans. R. Soc. A-Math. Phys. Eng. Sci. 369 (1948), 3136-3149 (2011).

7. T. Kimura, Y. Otani and J. Hamrle, Physical Review Letters 96 (3), 037201-037204 (2006).

8. T. Yang, T. Kimura and Y. Otani, Nature Physics 4 (11), 851 (2008).

9. I. Mihai Miron, G. Gaudin, S. Auffret, B. Rodmacq, A. Schuhl, S. Pizzini, J. Vogel and P. Gambardella, Nature Materials 9 (3), 230-234 (2010).

10. I. M. Miron, K. Garello, G. Gaudin, P.-J. Zermatten, M. V. Costache, S. Auffret, S. Bandiera, B. Rodmacq, A. Schuhl and P. Gambardella, Nature 476 (7359), 189-193 (2011).

11. L. Liu, O. J. Lee, T. J. Gudmundsen, D. C. Ralph and R. A. Buhrman, Physical Review Letters 109 (9), 096602 (2012).

12. L. Liu, C.-F. Pai, Y. Li, H. W. Tseng, D. C. Ralph and R. A. Buhrman, Science 336 (6081), 555 (2012).

13. C. Stamm, C. Murer, M. Berritta, J. Feng, M. Gabureac, P. M. Oppeneer and P. Gambardella, Physical Review Letters 119 (8), 087203 (2017).

14. G. Mihajlović, O. Mosendz, L. Wan, N. Smith, Y. Choi, Y. Wang and J. A. Katine, Applied Physics Letters 109 (19), 192404 (2016).

15. X. Fan, J. Wu, Y. Chen, M. J. Jerry, H. Zhang and J. Q. Xiao, Nature Communications 4 (1), 1799 (2013).

16. X. Fan, H. Celik, J. Wu, C. Ni, K.-J. Lee, V. O. Lorenz and J. Q. Xiao, Nature Communications 5 (1), 3042 (2014).

17. O. Fruchart and A. Thiaville, C. R. Phys. 6 (9), 921-933 (2005).

18. P. S. Keatley, V. V. Kruglyak, A. Neudert, E. A. Galaktionov, R. J. Hicken, J. R. Childress and J. A. Katine, Physical Review B 78 (21), 214412 (2008).

19. T. M. Spicer, P. S. Keatley, T. H. J. Loughran, M. Dvornik, A. A. Awad, P. Dürrenfeld, A. Houshang, M. Ranjbar, J. Åkerman, V. V. Kruglyak, et al., Physical Review B 98 (21), 214438 (2018).

20. J. Yoon, S.-W. Lee, J. H. Kwon, J. M. Lee, J. Son, X. Qiu, K.-J. Lee and H. Yang, Science Advances 3 (4) (2017).

21. K. Ishibashi, S. lihama, Y. Takeuchi, K. Furuya, S. Kanai, S. Fukami and S. Mizukami, Applied Physics Letters 117 (12), 122403 (2020).

22. Jhuria et. al. arXiv:1912.01377 [physics.app-ph] 2020

23. M. Baumgartner, K. Garello, J. Mendil, C. O. Avci, E. Grimaldi, C. Murer, J. Feng, M. Gabureac, C. Stamm, Y. Acremann, et al., Nature Nanotechnology 12 (10), 980-986 (2017).

24. P. S. Keatley, T. H. J. Loughran, E. Hendry, W. L. Barnes, R. J. Hicken, J. R. Childress and J. A. Katine, Review of Scientific Instruments 88 (12), 14 (2017).

25. C. H. Back, J. Heidmann and J. McCord, IEEE Transactions on Magnetics 35 (2), 637-642 (1999).

26. P. S. Keatley, P. Gangmei, M. Dvornik, R. J. Hicken, J. R. Childress and J. A. Katine, Applied Physics Letters 98 (8), 082506 (2011).

27. S. Karimeddiny, J. A. Mittelstaedt, R. A. Buhrman and D. C. Ralph, Physical Review Applied 14 (2), 024024 (2020). 
28. A. A. Tulapurkar, Y. Suzuki, A. Fukushima, H. Kubota, H. Maehara, K. Tsunekawa, D. D. Djayaprawira, N. Watanabe and S. Yuasa, Nature 438 (7066), 339-342 (2005).

29. P. Androvitsaneas, 'Quasi-static measurements for extraction of spin orbit torques' ArXiv

2020

30. L. Liu, T. Moriyama, D. C. Ralph and R. A. Buhrman, Physical Review Letters 106 (3), 036601 (2011). 